\title{
Textural features and SUV-based variables assessed by dual time point 18F-FDG PET/CT in locally advanced breast cancer
}

\author{
Ana María Garcia-Vicente ${ }^{1} \cdot$ David Molina $^{2} \cdot$ Julián Pérez-Beteta $^{2}$. \\ Mariano Amo-Salas ${ }^{3}$ Alicia Martínez-González ${ }^{2}$ Gloria Bueno ${ }^{4}$. \\ María Jesús Tello-Galán ${ }^{1} \cdot$ Ángel Soriano-Castrejón ${ }^{1}$
}

Received: 17 June 2017/ Accepted: 30 August 2017/Published online: 8 September 2017

(c) The Author(s) 2017. This article is an open access publication

\begin{abstract}
Aim To study the influence of dual time point $18 \mathrm{~F}-\mathrm{FDG}$ PET/CT in textural features and SUV-based variables and their relation among them.

Methods Fifty-six patients with locally advanced breast cancer (LABC) were prospectively included. All of them underwent a standard 18F-FDG PET/CT (PET-1) and a delayed acquisition (PET-2). After segmentation, SUV variables (SUVmax, SUVmean, and SUVpeak), metabolic tumor volume (MTV), and total lesion glycolysis (TLG) were obtained. Eighteen three-dimensional (3D) textural measures were computed including: run-length matrices (RLM) features, co-occurrence matrices (CM) features, and energies. Differences between all PET-derived variables obtained in PET-1 and PET-2 were studied.

Results Significant differences were found between the SUV-based parameters and MTV obtained in the dual time point PET/CT, with higher values of SUV-based variables and lower MTV in the PET-2 with respect to the PET-1. In relation with the textural parameters obtained in dual time point acquisition, significant differences were found for the short run emphasis, low gray-level run emphasis, short run high gray-level emphasis, run percentage, long run
\end{abstract}

Ana María Garcia-Vicente

angarvice@yahoo.es

1 Nuclear Medicine Department, University General Hospital, C/Obispo Rafael Torija s/n. 13005, Ciudad Real, Spain

2 Mathematical Oncology Laboratory (MôLAB), Universidad de Castilla-La Mancha, Ciudad Real, Spain

3 Department of Mathematics, University of Castilla-La Mancha, Ciudad Real, Spain

4 VISILAB Group, School of Industrial Engineering, Universidad de Castilla-La Mancha, Ciudad Real, Spain emphasis, gray-level non-uniformity, homogeneity, and dissimilarity. Textural variables showed relations with MTV and TLG.

Conclusion Significant differences of textural features were found in dual time point 18F-FDG PET/CT. Thus, a dynamic behavior of metabolic characteristics should be expected, with higher heterogeneity in delayed PET acquisition compared with the standard PET. A greater heterogeneity was found in bigger tumors.

Keywords Dual time point 18F-FDG PET/CT - Breast cancer - Tumor heterogeneity - Textural features

\section{Introduction}

Tumors are heterogeneous mixtures of cells, which differ in their morphology, genetics, and biological behavior. This complexity may underlie the inability of current therapies to significantly impact patient outcome [1]. Measuring tumor heterogeneity is not simple, since cellular diagnostic techniques, such as biopsies, are invasive and do not represent the full extent of genotypic and phenotypic tumor variations. Although intratumoral heterogeneity occurs at very small spatial scales, its macroscopic signatures can be observed using diagnostic imaging techniques. The advantages of using imaging techniques rely on the fact that they are non-invasive and take into account the whole tumor $[2,3]$.

The term 'textural analysis' refers to a variety of mathematical methods for quantifying the spatial distribution of voxel intensities in images [3, 4]. Those methods allow for an objective evaluation of the visible tumor properties, including heterogeneity. Many different textural analysis methods have been developed over the recent 
decades and used to define imaging biomarkers of relevance in oncology, named as "radiomics" [3].

Specifically in breast cancer, tumor metabolism assessed by 18 F-FDG PET/CT has shown multiple relations with immunohistochemical and histopathological factors [5-7]. Based on PET which reflects the tumor's biology, it is expected to provide substantial information about biological heterogeneity [4].

The two most common approaches to measure heterogeneity in PET are non-spatial methods (NSMs) and spatial textural methods (STMs). NSMs are based on the analysis of histograms constructed using the standard uptake value (SUV), and do not take into account any spatial information $[8,9]$. On the contrary, STMs use the spatial distribution of SUV in their computations. According to the type of spatial dependence, STMs can be divided in local and regional methods. Local STMs describe relationships between pairs of voxels within the tumor. The most commonly family of methods used is based on the so-called cooccurrence matrices (CMs) [10]. Regional STMs consider groups of voxels with the same intensity as connected volumes. Examples of those methods are those based on the so-called run-length matrices (RLMs) [11].

Based on the fact that a higher glycolytic activity of tumor tissues occurs between 3 to $5 \mathrm{~h}$ after administration of $18 \mathrm{~F}-\mathrm{FDG}$, dual time point acquisition has been used to improve tumor detectability and optimized the characterization of breast lesions [12, 13]. However, although the evolution in time of tumor activity metabolic variables, as SUV, has been described in the literature [9], no previous study has assessed the changes in texture parameters in a dual time point acquisition. Moreover, tumor volume is a conditioner for obtaining textural information [14].

Based on the limited reported evidence, the aim of this work was to study the differences of local and regional STMs textural features and SUV-based variables obtained in a dual time point 18F-FDG PET/CT and their relations, in patients with breast cancer.

\section{Materials and methods}

\section{Patients}

All reported patients were participants of an ongoing prospective study. The study was approved by the Institutional Review Board and written informed consent was obtained from all patients.

The inclusion criteria for our study were: (1) newly diagnosed breast cancer with clinical indication of neoadjuvant chemotherapy (NC), (2) lesion uptake higher than background, (3) absence of distant metastases confirmed by other methods previous to the request of the PET/CT for staging, and (4) breast lesion size of at least $2 \mathrm{~cm}$.

\section{FDG PET/CT acquisition}

All PET/CT examinations were performed on the same dedicated whole-body PET/CT machine (Discovery DSTE16s, GE Medical Systems) in three-dimensional (3D) mode. The first examination was performed $60 \mathrm{~min}$ after intravenous administration of approximately $370 \mathrm{MBq}$ of 18F-FDG (PET-1). The second examination was performed $3 \mathrm{~h}$ after injection, with a mean time of 127 min between the two phases (PET-2, range 112-138 min). Both acquisitions were performed following a standardized protocol [5].

The image voxel size was $5.47 \mathrm{~mm} \times 5.47 \mathrm{~mm} \times$ $3.27 \mathrm{~mm}$ with a slice thickness of $3.27 \mathrm{~mm}$ and no gap between slices. Matrix size was $128 \times 128$.

\section{Image analysis}

PET images in DICOM (Digital Imaging and Communication in Medicine) files were imported into the scientific software package Matlab (R2015b, The MathWorks, Inc., Natick, MA, USA) and pre-processed using in-house semiautomatic image segmentation software. The tumor was first manually located in a 3D box and then automatically segmented in three dimensions. Then, metabolic parameters were obtained as SUV max, SUVmean, SUVpeak, metabolic tumor volume (MTV), and total lesion glycolysis (TLG).

SUVmax is defined as the maximum uptake value in the segmented tumor, which reflects maximum tissue concentration of FDG in the volume of interest (VOI). SUVmean reflects the average uptake value in the VOI. SUVpeak is computed as the maximum average SUV taking a cube of $3 \times 3 \times 3$ voxels in the VOI. MTV is the volume of the VOI after segmentation. TLG is calculated as the product of SUVmean by MTV.

The formula used for the SUV computations was as follows:

$\mathrm{SUV}=\frac{\mathrm{SV} \cdot \mathrm{RS} \cdot W}{(\mathrm{RTD} \cdot \mathrm{DF}) \cdot e^{\left(-\mathrm{Ln}(2) \cdot \frac{\mathrm{Et}}{\mathrm{HF}}\right)}}$,

where $\mathrm{SV}$ is the stored value, RS the rescaled slope, $W$ is the patient weight, RTD is the radiopharmaceutical injected dose and HF its half-life, DF is the decay factor, and Et is the elapsed time for each slice processed. This formula was selected as it allows comparing raw PET-1 and PET-2 data, since it takes into account the elapsed time from dose injection. 
The regions in the 3D box equal to or above $40 \%$ the SUVmax were selected to automatically delineate the volume of interest (VOI). In case of central hypometabolism and a metabolic activity below the selected threshold value, this volume was considered as necrosis and excluded from the volume assessment. In case of multiple breast lesions (multicenter or multifocal cancer), those with the highest FDG uptake were selected for the analysis.
A set of 18 3D textural features was automatically computed using the Matlab software [15]. These measures provide a (local or regional) characterization of the spatial relations between voxels within the tumour. Our choice of textural measures is listed in Table 1. RLM characterizes large areas within the tumor (groups of voxels) to provide information of regional heterogeneity [11]. Each cell in RLMs $(i, j)$ was computed as the number of runs of length $j$
Table 1 Definition of the heterogeneity measures computed in this study

\begin{tabular}{|c|c|c|}
\hline Type of measure & Name & Formula \\
\hline Co-occurrence matrix & Entropy (ENT) & $-\sum_{i=1}^{N} \sum_{j=1}^{N} \mathrm{CM}(i, j) \cdot \ln [\mathrm{CM}(i, j)]$ \\
\hline Co-occurrence matrix & Homogeneity (HOM) & $\sum_{i=1}^{N} \sum_{j=1}^{N} \frac{\mathrm{CM}(i, j)}{1+(i-j)^{2}}$ \\
\hline Co-occurrence matrix & Contrast $(\mathrm{CON})$ & $\sum_{i=1}^{N} \sum_{j=1}^{N} \mathrm{CM}(i, j) \cdot(i-j)^{2}$ \\
\hline Co-occurrence matrix & Dissimilarity (DIS) & $\sum_{i=1}^{N} \sum_{j=1}^{N} \mathrm{CM}(i, j) \cdot|i-j|$ \\
\hline Co-occurrence matrix & Uniformity (UNI) & $\sum_{i=1}^{N} \sum_{j=1}^{N}[\mathrm{CM}(i, j)]^{2}$ \\
\hline Run-length matrix & Long run emphasis (LRE) & $\frac{1}{n_{r}} \sum_{i=1}^{N} \sum_{j=1}^{M} \operatorname{RLM}(i, j) \cdot j^{2}$ \\
\hline Run-length matrix & Short run emphasis (SRE) & $\frac{1}{n_{r}} \sum_{i=1}^{N} \sum_{j=1}^{M} \frac{R L M(i, j)}{j^{2}}$ \\
\hline Run-length matrix & Low gray-level run emphasis (LGRE) & $\frac{1}{n_{r}} \sum_{i=1}^{N} \sum_{j=1}^{M} \frac{\mathrm{RLM}(i, j)}{i^{2}}$ \\
\hline Run-length matrix & High gray-level run emphasis (HGRE) & $\frac{1}{n_{r}} \sum_{i=1}^{N} \sum_{j=1}^{M} \operatorname{RLM}(i, j) \cdot i^{2}$ \\
\hline Run-length matrix & Short run low gray-level emphasis (SRLRE) & $\frac{1}{n_{r}} \sum_{i=1}^{N} \sum_{j=1}^{M} \frac{\operatorname{RLM}(i, j)}{i^{2} \cdot j^{2}}$ \\
\hline Run-length matrix & Short run high gray-level emphasis (SRHGE) & $\frac{1}{n_{r}} \sum_{i=1}^{N} \sum_{j=1}^{M} \frac{\operatorname{RLM}(i, j) \cdot i^{2}}{j^{2}}$ \\
\hline Run-length matrix & Long run low gray-level emphasis (LRLGE) & $\frac{1}{n_{r}} \sum_{i=1}^{N} \sum_{j=1}^{M} \frac{\operatorname{RLM}(i, j) \cdot j^{2}}{i^{2}}$ \\
\hline Run-length matrix & Long run high gray-level emphasis (LRHGE) & $\frac{1}{n_{r}} \sum_{i=1}^{N} \sum_{j=1}^{M} \operatorname{RLM}(i, j) \cdot i^{2} \cdot j^{2}$ \\
\hline Run-length matrix & Gray-level non-uniformity (GLNU) & $\frac{1}{n_{r}} \sum_{i=1}^{N}\left(\sum_{j=1}^{M} \operatorname{RLM}(i, j)\right)^{2}$ \\
\hline Run-length matrix & Run-length non-uniformity (RLNU) & $\frac{1}{n_{r}} \sum_{j=1}^{M}\left(\sum_{i=1}^{N} \operatorname{RLM}(i, j)\right)^{2}$ \\
\hline Run-length matrix & Run percentage (RPC) & $\frac{n_{r}}{\sum_{i=1}^{N} \sum_{j=1}^{M} \operatorname{RLM}(i, j) \cdot j}$ \\
\hline Energy & Specific energy (SE) & $\frac{\left(\int|\nabla u|^{2} \mathrm{~d} V\right)^{\frac{1}{2}}}{\left(\int|u|^{2} \mathrm{~d} V\right)^{\frac{1}{2}}}$ \\
\hline Energy & Total p-energy (TE) & $\frac{\left(\int|\nabla u|^{2} \mathrm{~d} V\right)^{\frac{1}{2}}}{\max _{g \in u}}$ \\
\hline
\end{tabular}

For CM measures, $C M(i, j)$ stands for the co-occurrence matrix, and $N$ is the number of classes of gray levels taken (in this study 16). For RLM measures, $R L M(i, j)$ is the run-length matrix, $n_{r}$ is the number of runs, $N$ is the number of classes of gray levels, and $M$ is the size in voxels of the largest region found 
formed by voxels of intensity in box $i$ in all the 13 possible directions in 3D.

The CMs describe the arrangements of pairs of elements (voxels) within 2D images [16]. As they measure only relations between two voxels at a time, they are usually considered to provide information on the local texture of images. Our CMs were constructed by including the relationships between voxels in all of the 13 possible directions in 3D [17, 18] taking only adjacent voxels. Thus, the relations with the 26 neighbours of each voxel in 3D were considered.

The energies are STMs based on absolute gradients obtained from SUV levels. They are computed as the sum of all the spatial SUV gradient variations within the segmented tumor. The spatial SUV gradient is a vector computed on every tumor voxel by computing the differences between the SUV values of the adjacent voxels in 3D. Two different energies were computed. The spatial energy (SE) is independent of $\mathrm{SUV}_{\max }$, as it is normalized by the norm of all the SUV levels within the tumor. This is an intensive variable measuring the level of variations in SUV per unit of volume and unit of SUV. The total energy (TE) is normalized by $\mathrm{SUV}_{\text {max }}$, accounting for the spatial variations of the SUV within its range of values. It is an extensive variable, since enlarging the domain leads to larger values of the quantity (see Table 1).

\section{Statistical analysis}

Statistical analysis was performed using SPSS software (v. 22.0.00 IBM, New York, NY, USA). Qualitative variables were summarized using percentages and frequencies, and quantitative variables using mean and standard deviation.

Spearman's correlation coefficient was considered to study the relation between textural and metabolic variables due to the non-parametric nature of the metabolic variables.

To study the repeated measures of textural variables, $T$ test was used for dependent samples and Wilcoxon test in the non-parametric case.

For statistical analysis, a categorical separation of lesions attending to their MTV was performed (group I: $\mathrm{MTV} \leq 10 \mathrm{~cm}^{3}$ and group II: MTV $>10 \mathrm{~cm}^{3}$ ).
Table 2 Metabolic tumor variables obtained in PET-1 and PET-2 and differences

\begin{tabular}{|c|c|c|c|}
\hline Metabolical variables & mean $\pm \mathrm{SD}(\mathrm{PET}-1)$ & mean $\pm \mathrm{SD}($ PET-2) & $\mathrm{T} / \mathrm{Z}(p$ value $)$ \\
\hline SUVmax & $9.16 \pm 5.76$ & $10.81 \pm 7.64$ & $-5.40(0.000)$ \\
\hline SUVpeak & $7.11 \pm 4.56$ & $8.36 \pm 6.11$ & $-5.13(0.000)$ \\
\hline SUVmean & $5.59 \pm 3.54$ & $6.60 \pm 4.60$ & $-5.88(0.000)$ \\
\hline MTV & $16.59 \pm 20.13$ & $15.50 \pm 18.93$ & $2.53(0.014)$ \\
\hline TLG & $110.26 \pm 209.62$ & $125.62 \pm 240.87$ & $0.36(0.721)$ \\
\hline ENT & $4.98 \pm 0.21$ & $4.96 \pm 0.26$ & $0.63(0.533)$ \\
\hline $\mathrm{HOM}$ & $0.23 \pm 0.04$ & $0.22 \pm 0.05$ & $2.42(0.019)$ \\
\hline $\mathrm{CON}$ & $28.05 \pm 10.09$ & $29.61 \pm 11.13$ & $-1.81(0.075)$ \\
\hline DIS & $4.12 \pm 0.83$ & $4.25 \pm 0.89$ & $-2.11(0.039)$ \\
\hline UNI & $0.01 \pm 0.003$ & $0.009 \pm 0.005$ & $-1.10(0.279)$ \\
\hline SRE & $0.62 \pm 0.08$ & $0.66 \pm 0.08$ & $-3.47(0.001)$ \\
\hline LRE & $14.33 \pm 24.89$ & $12.70 \pm 27.25$ & $2.45(0.014)$ \\
\hline LGRE & $0.19 \pm 0.04$ & $0.18 \pm 0.04$ & $2.38(0.021)$ \\
\hline HGRE & $53.55 \pm 8.75$ & $55.28 \pm 8.96$ & $-1.77(0.083)$ \\
\hline SRLGE & $0.12 \pm 0.04$ & $0.12 \pm 0.03$ & $1.48(0.721)$ \\
\hline SRHGE & $32.91 \pm 9.50$ & $36.66 \pm 8.50$ & $-3.03(0.004)$ \\
\hline LRLGE & $2.68 \pm 5.43$ & $3.35 \pm 10.95$ & $-0.58(0.561)$ \\
\hline LRHGE & $584.87 \pm 750.78$ & $458.70 \pm 473.51$ & $-2.55(0.011)$ \\
\hline GLNU & $5.82 \pm 4.57$ & $5.48 \pm 4.38$ & $-1.98(0.047)$ \\
\hline RLNU & $22.95 \pm 15.48$ & $24.04 \pm 16.63$ & $-1.09(0.281)$ \\
\hline RPC & $0.49 \pm 0.12$ & $0.53 \pm 0.13$ & $-4.25(0.000)$ \\
\hline SE & $0.12 \pm 0.03$ & $0.13 \pm 0.02$ & $-2.44(0.018)$ \\
\hline TE & $8.34 \pm 2.41$ & $8.11 \pm 2.40$ & $1.56(0.125)$ \\
\hline
\end{tabular}

A significance level of $p$ value $<0.05$ was used in all statistical tests. Correlation coefficient values over 0.75 were taken as indicators of strong correlation

$S D$ standard deviation, $S U V$ standard uptake value, $M T V$ metabolic tumor volume, $T L G$ total lesion glycolysis, $T$ value obtained from $T$ test for dependent samples, $Z$ value obtained from Wilcoxon test in the non-parametric case. A negative T/Z value means that the value obtained in PET-1 was lower that its correspondent value in PET-2 
Table 3 Relations of volume-based and textural parameters obtained in PET-1 and PET-2

\begin{tabular}{|c|c|c|c|c|}
\hline Textural variables & MTV (PET-1) r ( $p$ value) & TLG (PET-1) r ( $p$ value) & MTV (PET-2) r ( $p$ value) & TLG (PET- 2$) r(p$ value $)$ \\
\hline ENT (PET-1) & $0.36(0.007)$ & $0.37(0.005)$ & $0.40(0.002)$ & $0.38(0.004)$ \\
\hline HOM (PET-1) & $0.83(0.000)$ & $0.62(0.000)$ & $0.67(0.000)$ & $0.46(0.000)$ \\
\hline CON (PET-1) & $-0.88(0.000)$ & $-0.69(0.000)$ & $-0.74(0.000)$ & $-0.55(0.000)$ \\
\hline DIS (PET-1) & $-0.88(0.000)$ & $-0.68(0.000)$ & $-0.74(0.000)$ & $-0.54(0.000)$ \\
\hline UNI (PET-1) & $-0.29(0.031)$ & $-0.36(0.006)$ & $-0.38(0.003)$ & $-0.41(0.002)$ \\
\hline SRE (PET-1) & $-0.52(0.000)$ & $-0.32(0.017)$ & $-0.37(0.005)$ & $-0.17(0.222)$ \\
\hline LRE (PET-1) & $0.91(0.000)$ & $0.68(0.000)$ & $0.76(0.000)$ & $0.51(0.000)$ \\
\hline LGRE (PET-1) & $0.05(0.700)$ & $-0.14(0.313)$ & $0.04(0.785)$ & $0.14(0.313)$ \\
\hline HGRE (PET-1) & $-0.53(0.000)$ & $-0.38(0.004)$ & $-0.42(0.001)$ & $-0.27(0.045)$ \\
\hline SRLGE (PET-1) & $-0.05(0.696)$ & $-0.07(0.613)$ & $0.002(0.787)$ & $-0.002(0.991)$ \\
\hline SRHGE (PET-1) & $-0.58(0.000)$ & $-0.39(0.003)$ & $-0.50(0.000)$ & $-0.30(0.027)$ \\
\hline LRLGE (PET-1) & $0.52(0.000)$ & $0.33(0.013)$ & $0.33(0.014)$ & $0.16(0.253)$ \\
\hline gLRHGE (PET-1) & $0.79(0.000)$ & $0.70(0.000)$ & $0.71(0.000)$ & $0.60(0.000)$ \\
\hline GLNU (PET-1) & $0.96(0.000)$ & $0.83(0.000)$ & $0.88(0.000)$ & $0.70(0.000)$ \\
\hline RLNU (PET-1) & $0.79(0.000)$ & $0.78(0.000)$ & $0.80(0.000)$ & $0.74(0.000)$ \\
\hline RPC (PET-1) & $-0.87(0.000)$ & $-0.63(0.000)$ & $-0.71(0.000)$ & $-0.46(0.000)$ \\
\hline SE (PET-1) & $-0.89(0.000)$ & $-0.70(0.000)$ & $-0.77(0.000)$ & $-0.56(0.000)$ \\
\hline TE (PET-1) & $0.83(0.000)$ & $0.81(0.000)$ & $0.79(0.000)$ & $0.72(0.000)$ \\
\hline HOM (PET-2) & $0.84(0.000)$ & $0.71(0.000)$ & $0.70(0.000)$ & $0.57(0.000)$ \\
\hline DIS (PET-2) & $-0.89(0.000)$ & $-0.79(0.000)$ & $-0.78(0.000)$ & $-0.66(0.000)$ \\
\hline SER (PET-2) & $-0.62(0.000)$ & $-0.52(0.000)$ & $-0.48(0.000)$ & $-0.37(0.000)$ \\
\hline LRE (PET-2) & $0.91(0.000)$ & $0.79(0.000)$ & $0.78(0.000)$ & $0.65(0.000)$ \\
\hline LGRE (PET-2) & $0.11(0.421)$ & $0.04(0.769)$ & $0.02(0.854)$ & $-0.06(0.648)$ \\
\hline SRHGE (PET-2) & $-0.73(0.000)$ & $-0.72(0.000)$ & $-0.65(0.000)$ & $-0.60(0.000)$ \\
\hline LRHGE (PET-2) & $0.73(0.000)$ & $0.78(0.000)$ & $0.76(0.000)$ & $0.75(0.000)$ \\
\hline GLNU (PET-2) & $0.91(0.000)$ & $0.84(0.000)$ & $0.89(0.000)$ & $0.75(0.000)$ \\
\hline RPC (PET-2) & $-0.88(0.000)$ & $-0.76(0.000)$ & $-0.75(0.000)$ & $-0.62(0.000)$ \\
\hline SE (PET-2) & $-0.86(0.000)$ & $-0.79(0.000)$ & $-0.82(0.000)$ & $-0.71(0.000)$ \\
\hline
\end{tabular}

A significance level of $p$ value $<0.05$ was used in all statistical tests. Correlation coefficient values over 0.75 were taken as indicators of strong correlation

A significance level of $p$ value $<0.05$ was used in all statistical test. This $p$ value was corrected when needed. Correlation coefficient values over 0.75 were taken as indicators of strong correlation.

\section{Results}

Fifty-six patients satisfied the inclusion criteria. The mean age \pm SD was $52.75 \pm 13.68$ years. Histologically, 53 tumors were ductal invasive and three were lobular invasive carcinomas.

Significant differences were found between the mean values of SUV-based parameters and MTV obtained in the dual time point PET/CT, with higher values of SUV-based variables and lower MTV in the PET-2 with respect to the PET-1. No significant differences were observed for the TLG.
As to the textural parameters, significant differences were found for the SRE (short run emphasis), LGRE (low gray-level run emphasis), SRHGE (short run high graylevel emphasis), LRHGE (long run high gray-level emphasis), RPC (run percentage), LRE (long run emphasis), GLNU (gray-level non-uniformity), HOM (homogeneity), DIS (dissimilarity), and SE which means that PET-2 showed, in general terms, larger heterogeneity than PET-1. The detailed results are shown in Table 2.

Most textural variables with significant changes in PET2 with respect to PET-1 showed lower values in PET-1. Only three textural variables (HOM, LRE, and LGRE) suffered a decrease in their values in PET-2.

We also studied the association between textural features on one side, and SUV- and volume-based variables on the other. Textural features were found to be associated only with volume-based variables (MTV and TLG). No SUV-based variable was significantly associated with 

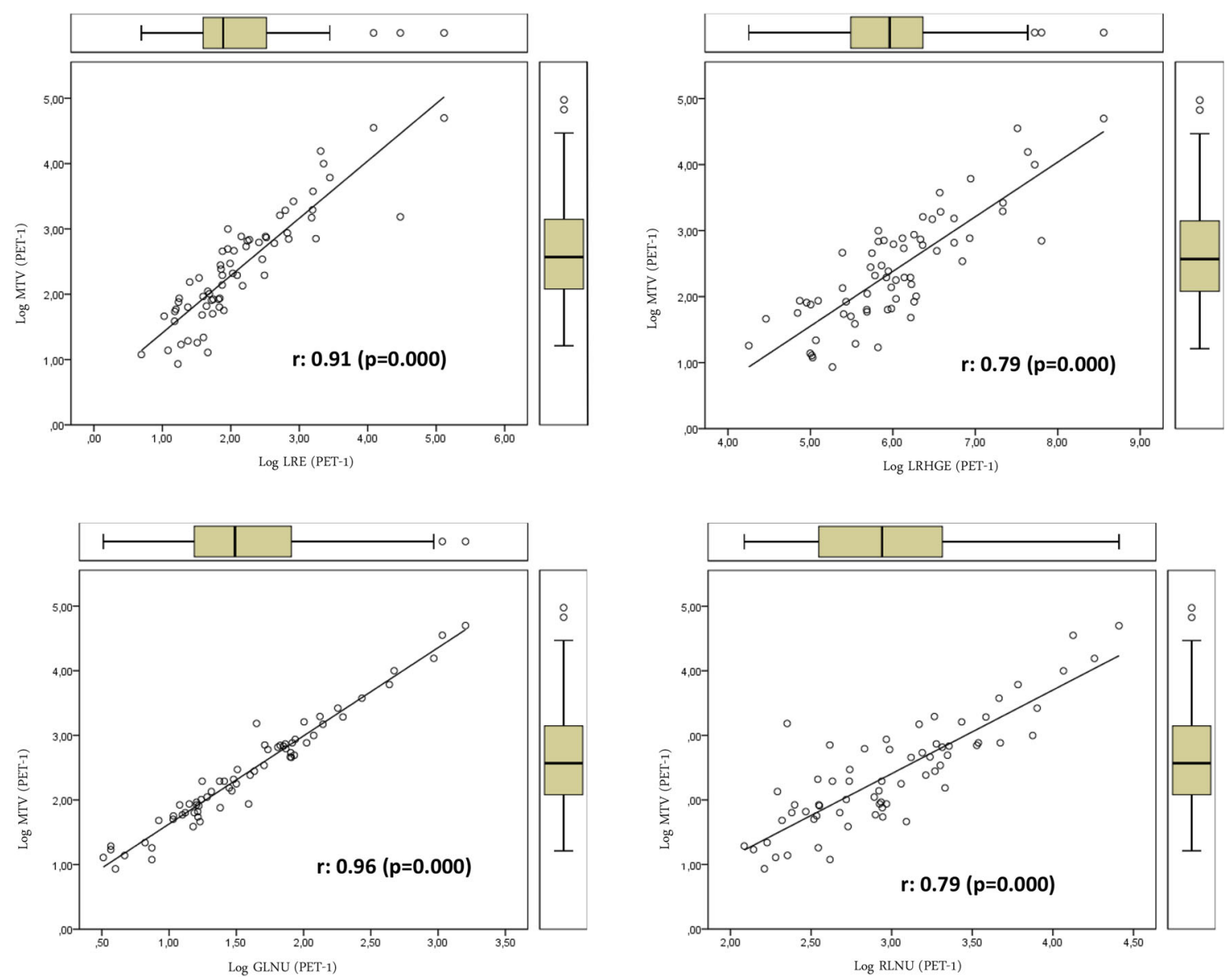

Fig. 1 Relations of textural parameters with the metabolic tumor volume obtained in PET-1 using the log scale that showed positive association with heterogeneity

textural parameters. Table 3 shows the relation between volume-based variables obtained from PET-1 and PET-2 and the textural variables of PET-1 and those that showed significant differences in the PET-2. TE, LRE, LRHGE, GLNU, RLNU, and HOM showed direct relations with MTV. On the contrary SE, RPC, ENT (entropy), CON (contrast), and DIS showed inverse relations with MTV (Figs. 1, 2). We found a more significant relation between textural variables obtained from PET-2 and the TLG value with direct and inverse associations (Figs. 3, 4; Table 3).

With regard to the parallel analysis, dividing the lesions into two groups attending to the MTV (group I: MTV $\leq 10 \mathrm{~cm}^{3}$ and group II: MTV $>10 \mathrm{~cm}^{3}$ ), significant relations were found between textural variables with MTV and TLG. Table 4 summarizes the most significant associations in PET-1 MTV. For MTV obtained in PET-2, a less number of significant and strong relations were found: group I [PET-2 SE, $r=-0.82(p<0.0001)$, PET-2 GLNU, $r=0.88,(p<0.0001)$ ] and group II [PET-2 GLNU, $r=0.80,(p<0.0001)]$.

Figure 5 represents gray-level distribution of voxels for texture analysis.

\section{Discussion}

Assessment of tumor textures, using quantitative features, has attracted much attention in the medical imaging research community. However, its use in clinical practice is not still widespread, probably due to the lack of standardized and validated methods [3].

PET reports the metabolic tumor cells' behavior and thus has interesting properties for imaging inference. It has been shown that FDG tumor uptake is not only related to an increased metabolic rate, but also to hypoxia, aggressiveness, and cell proliferation [19].

Many ways to quantify tumor's heterogeneity are available [3]. The most used ones are the histogram-based features and the STMs. Histogram-based features rely on the global computation of tumor heterogeneity taking into account only the SUV values and not the spatial relations between voxels within the tumor. Since we were interested in retaining the spatial information, we used only STMs in this work to characterize the spatial heterogeneity.

Dual time point 18F-FDG PET/CT has been previously used to assess the variations of SUV-based parameters 

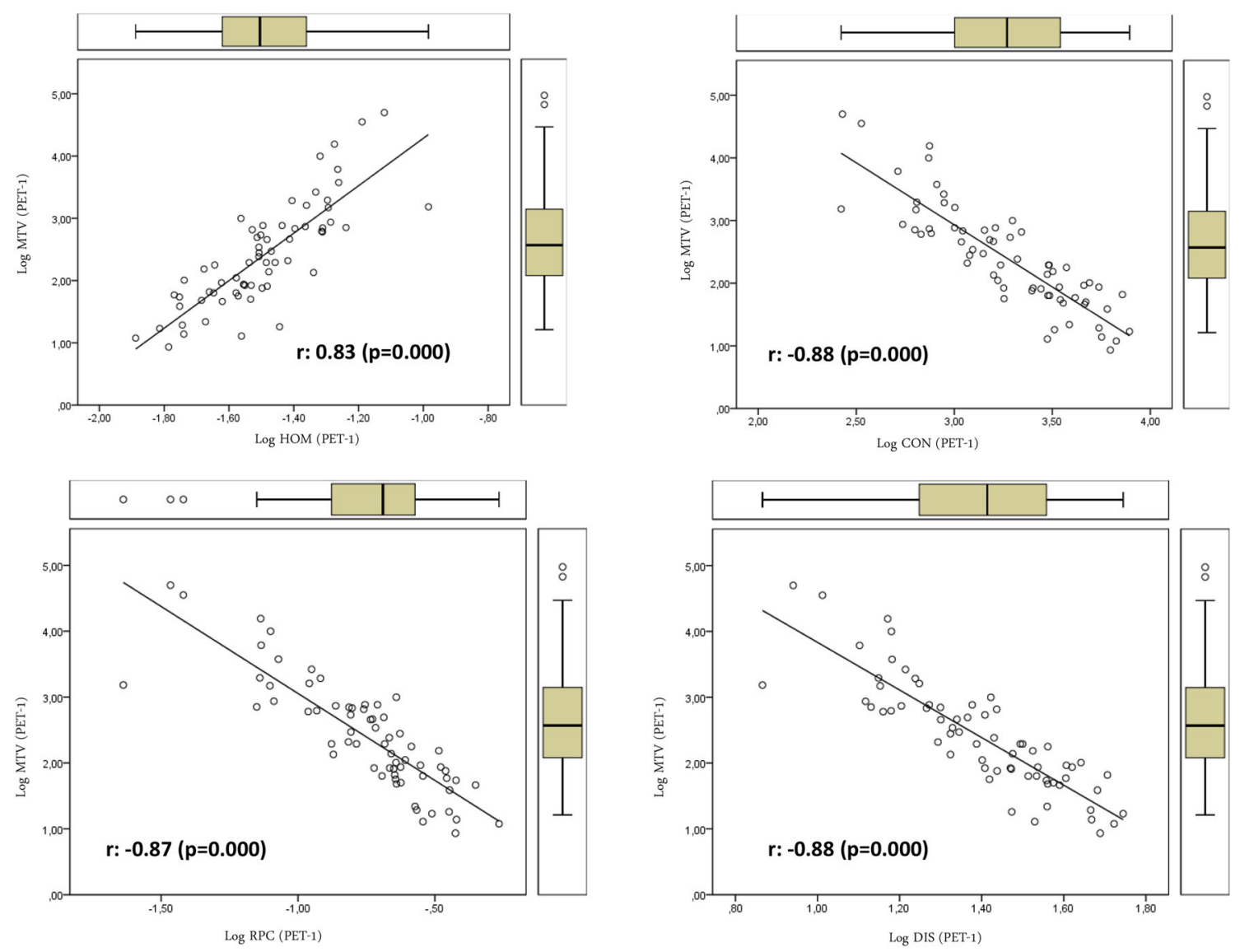

Fig. 2 Relations of textural parameters with the metabolic tumor volume obtained in PET-1 $\mathrm{t}$ using the log scale hat showed negative association with heterogeneity

$[12,13,20,21]$. However, to our knowledge, this is the first work assessing the differences between textural features in a dual time point acquisition. We observed that several textural variables quantifying tumor heterogeneity showed significant increases in the delayed PET as compared to the standard PET acquisition. Mena et al. [22], using dual time point 18F-FDG PET/CT in patients with pancreatic adenocarcinoma, reported changes greater than $10 \%$ of the tumor heterogeneity index in $40.8 \%$ of tumors at delayed imaging, when gradient segmentation method was used. However, stable metabolic intratumoral heterogeneity values were seen between early and delayed PET when using threshold segmentation method.

Textural features did not show high correlation with SUVmax, SUVpeak, and SUVmean. This means that an isolated semiquantitative measure, as SUV, does not provide a prediction of the total tumor metabolical distribution. Thus, small areas of homogeneous uptake can have high or low SUVmax, whereas both larger homogeneous or heterogeneous lesions can exhibit a wide range of maximum uptakes.

On the other hand, some of the textural parameters studied showed correlation with MTV and TLG. This is easy to explain, since tumor volume is important when computing textural features. For instance, larger tumors may have larger connected regions and provide larger values of the RLM-based variables.

In the present work, textural variables showed direct and inverse relations with MTV, with independence of the segmented tumor volume. The different meaning and robustness of each textural variable with regard to heterogeneity information could explain our results. We also found a more significant relation of textural variables obtained from PET-2 with the TLG values. This may be related to the high dependence of some textural variables on the gray-level intensity and the close relation of TLG with tumor metabolism.

The previous works have reported that intratumor heterogeneity increases as tumors grow [14, 23, 25]. This may be due to the fact that larger tumors have more potential to be composed by several different types of tissues and regions with variable uptake. Smaller tumors may also have heterogeneity at the cellular and tissue levels, but it may be blurred in PET images due to the limited spatial resolution.

Several authors have addressed the tumor volume confounding effect, finding that the correlation between 

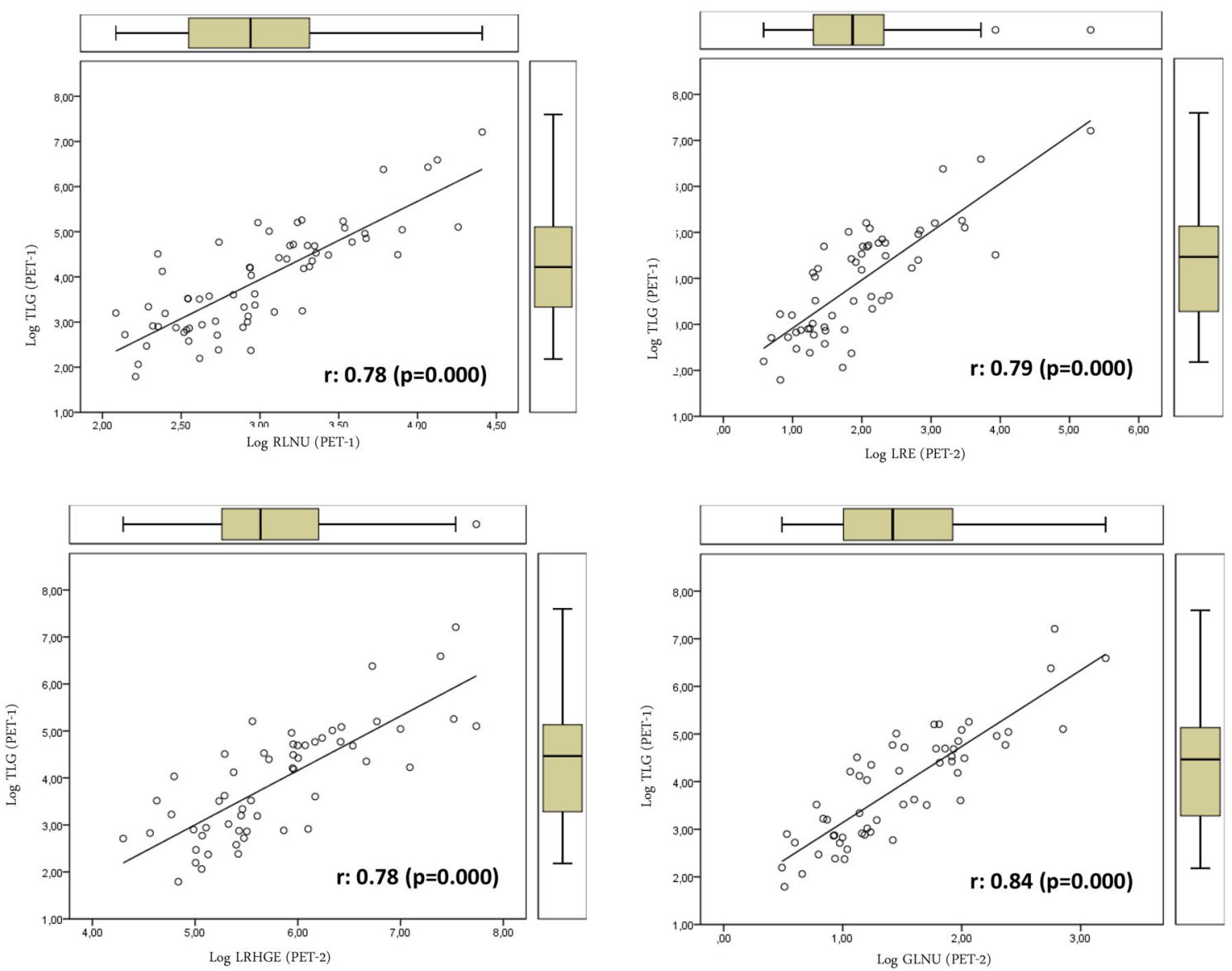

Fig. 3 Relations of textural parameters with total lesion glycolysis obtained in PET-1 and PET-2 using the log scale that showed positive association with heterogeneity
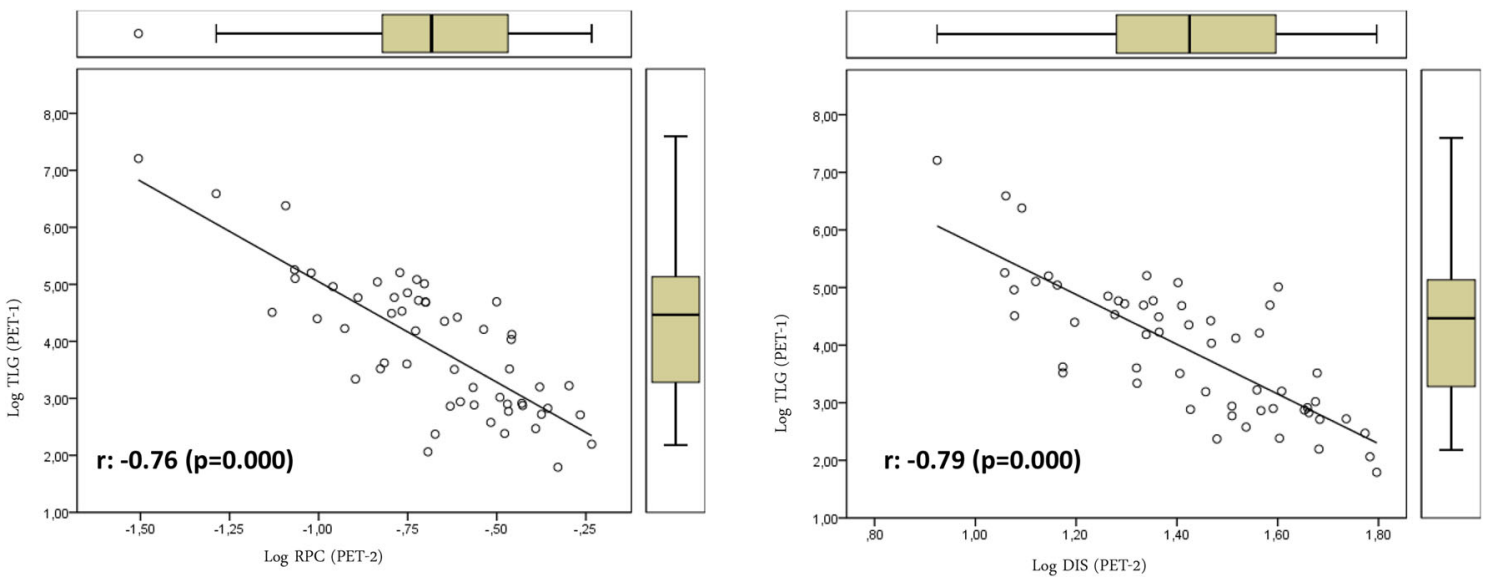

Fig. 4 Relations of textural parameters with total lesion glycolysis obtained in PET-2 using the log scale that showed negative association with heterogeneity

textural features with MTV tends to decrease with increasing volumes. Thus, volume and heterogeneity might offer a complementary information [26].

Although there is not a consensus about the optimal tumor volume allows assessing correctly textural variables, most studies using textural features have considered volumes greater than $3-5 \mathrm{~cm}^{3}$, based on PET cannot characterize heterogeneity in smaller volumes because of its limited spatial resolution [23-25].

In the present work, we also used the energies as textural variables. These measures are novel robust features accounting for tumor heterogeneity. They have many 
Table 4 Relations of volume-based and textural parameters dividing lesions into two groups (group I: MTV $\leq 10 \mathrm{~cm}^{3}$ and group II: $\mathrm{MTV}>10 \mathrm{~cm}^{3}$ in PET-1)

\begin{tabular}{llll}
\hline Textural variables & MTV $\leq 10 \mathrm{~cm}^{3}($ PET- $) \mathrm{r}(p$ value $)$ & Textural variables & MTV $>10 \mathrm{~cm}^{3}($ PET-1) $\mathrm{r}(p$ value $)$ \\
\hline GLNU (PET-1) & $0.86(0.000)$ & LRE (PET-1) & $0.84(0.000)$ \\
SE (PET-2) & $0.75(0.000)$ & GLNU (PET-1) & $0.87(0.000)$ \\
GLNU (PET-2) & $0.76(0.000)$ & LRE (PET-2) & $0.75(0.000)$ \\
\hline
\end{tabular}
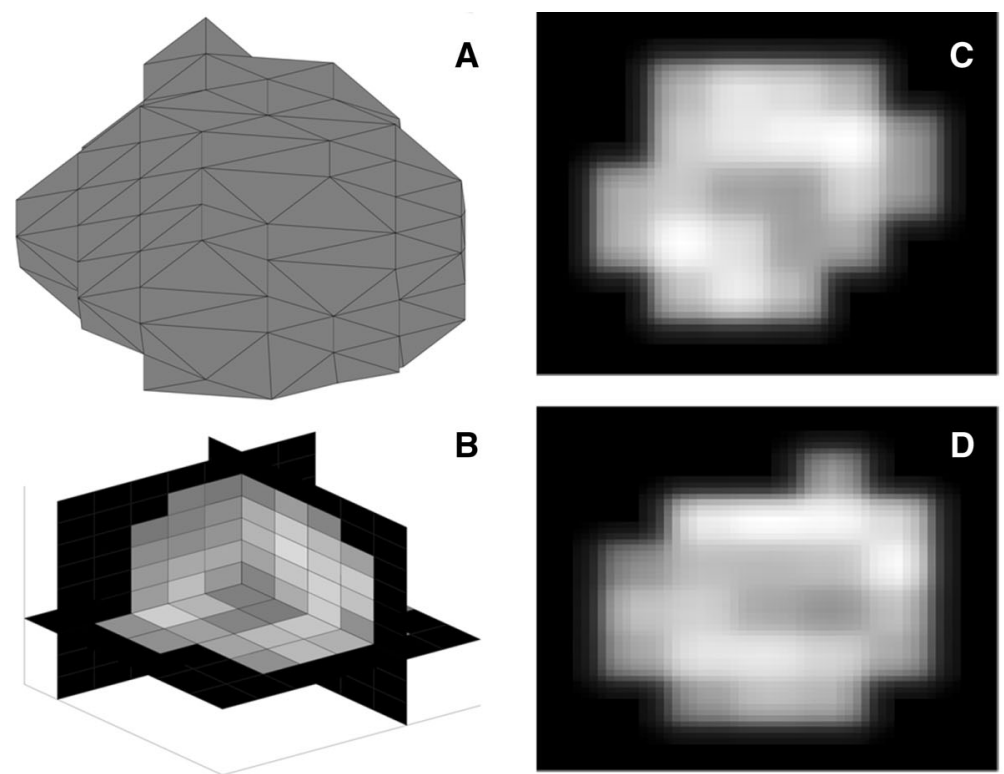
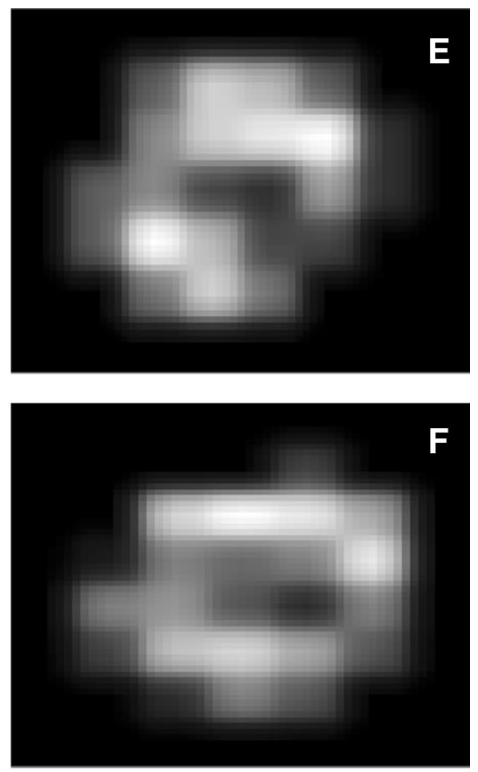

Fig. 5 Breast tumor segmentation (a) and voxel representation in 3D image reconstruction (b). Raw gray level distribution in PET-1 (c) and PET-2 (d) used for energy analysis. e and $\mathbf{f}$ show gray-level distribution after discretization of the $\mathbf{c}$ and $\mathbf{d}$

advantages over RLM- and CM-based variables. First, they are independent of the choice of the dynamic range. Second, they have a well-defined limit as the number of voxels increases. Finally, they provide a combination of local information, because of the use of gradients, and global, because of the integral averaging over the whole tumor. These measures could be a relevant addition to the standard radiomic toolbox with fewer limitations than RLM- and CM-based variables [27].

The previous works have addressed the association between textural features and SUV-derived biological variables in $\operatorname{LABC}[19,28]$. However, to our knowledge, no study has assessed the heterogeneity in a dual time point PET. Moreover, due to the limited reported evidence and the discrepancies in the literature, further analysis of heterogeneity in LABC is in order.

With respect to the limitations, textural features robustness has been put into question, basically due to questions of interpretation and even the methodology of its obtaining [22, 27-29]. However, their use is still widespread, and therefore, more studies are needed to validate their underlying characteristics.
The main strength of this work is that it is the first reported study of the evolution in time of textural variables in breast cancer assessed in a dual time PET/CT acquisition, addressing that texture is dynamic, as SUV-based variables are.

\section{Conclusions}

Significant differences between textural features were found in the dual time point 18F-FDG PET/CT. A dynamic behavior of metabolic characteristics was observed, with a higher heterogeneity in delayed PET acquisition compared with the obtained one in the standard PET.

Textural features were related to tumor volume, with higher heterogeneity for bigger tumors.

Acknowledgements This work has been supported by Ministerio de Economía y Competitividad/FEDER, Spain [Grant Number MTM2015-71200-R] and Consejería de Educación Cultura y Deporte from Junta de Comunidades de Castilla-La Mancha, Spain [Grant Number PEII-2014-031-P]. 
Open Access This article is distributed under the terms of the Creative Commons Attribution 4.0 International License (http://crea tivecommons.org/licenses/by/4.0/), which permits unrestricted use, distribution, and reproduction in any medium, provided you give appropriate credit to the original author(s) and the source, provide a link to the Creative Commons license, and indicate if changes were made.

\section{References}

1. Fischer R, Pusztai L, Swanton C. Cancer heterogeneity: implications for targeted therapeutics. Br J Cancer. 2013;108:479-85.

2. Davnall F, Yip CS, Ljungqvist G, Selmi M, Ng F, Sanghera B, et al. Assessment of tumor heterogeneity: an emerging imaging tool for clinical practice? Insights Imaging. 2012;3:573-89.

3. Alic L, Niessen WJ, Veenland JF. Quantification of heterogeneity as a biomarker in tumor imaging: a systematic review. PLoS One. 2014;9(10):e110300.

4. Moscoso A, Aguiar P, Pardo-Montero J, Ruibal A. Textural analysis to assess heterogeneity in breast cancer. Biomark J. 2016;2:1-12.

5. García-Vicente AM, Soriano-Castrejón A, León-Martín A, Chacón-López-Muñiz I, Muñoz-Madero V, Muñoz-Sánchez MM, et al. Molecular subtypes of breast cancer: metabolic correlation with ${ }^{18}$ F-FDG PET/CT. Eur $J$ Nucl Med Molec Imag. 2013;40:1304-11.

6. Bolouri MS, Elias SG, Wisner DJ, Behr SC, Hawkins RA, Suzuki $\mathrm{SA}$, et al. Triple-negative and non-triple-negative invasive breast cancer: association between $\mathrm{MR}$ and fluorine 18 fluorodeoxyglucose PET imaging. Radiology. 2013;269:354-61.

7. Koo HR, Park JS, Kang KW, Cho N, Chang JM, Bae MS, et al. 18F-FDG uptake in breast cancer correlates with immunohistochemically defined subtypes. Eur Radiol. 2014;24:610-8.

8. Chicklore S, Goh V, Siddique M, Roy A, Marsden PK, Cook GJR. Quantifying tumour heterogeneity in 18F-FDG PET/CT imaging by texture analysis. Eur J Nucl Med Mol Imaging. 2013;40:133-40.

9. Burger AI, Vargas HA, Apte A, Beattie BJ, Humm JL, Gonen M, et al. PET quantification with a histogram derived total activity metric: superior quantitative consistency compared to total lesion glycolysis with absolute or relative SUV thresholds in phantoms and lung cancer patients. Nucl Med Bio. 2014;41:410-8.

10. Haralick RM, Shanmugam K, Dinstein I. Textural features of image classification. IEEE Trans Syst Man Cyber. 1973;3:610-21.

11. Galloway MM. Texture analysis using gray level run lengths. Comput Graph Image Process. 1975;4:172-9.

12. Mavi A, Urhan M, Yu JQ, Zhuang H, Houseni M, Cermik TF, et al. Dual time point $18 \mathrm{~F}-\mathrm{FDG}$ PET imaging detects breast cancer with high sensitivity and correlates well with histologic subtypes. J Nucl Med. 2006;47:1440-6.

13. Zytoon AA, Murakami K, El-Kholy M-R, El-Shorbagy E. Dual time point FDG-PET/CT imaging. Potential tool for diagnosis of breast cancer. Clin Radiol. 2008;63:1213-27.

14. Orlhac F, Soussan M, Maisonobe JA, Garcia CA, Vanderlinden B, Buvat I. Tumor texture analysis in 18F-FDG PET: relationships between texture parameters, histogram indices, standardized uptake values, metabolic volumes, and total lesion glycolysis. J Nucl Med. 2014;55:414-22.

15. Xu D, Kurani AS, Furst JD, Raicu DS. Run-length encoding for volumetric texture. In: The 4th IASTED international conference on visualization, imaging, and image processing. 2004. pp. $452-8$.

16. Li LM, Castellano C, Bonilha L, Cendes F. Texture analysis of medical images. Clin Radiol. 2004;59:1061-9.

17. Tixier F, Le Rest CC, Hat M, Albarghach N, Pradier O, Metges $\mathrm{JP}$, et al. Intratumour heterogeneity characterized by textural features on baseline 18F-FDG PET images predicts response to concomitant radiochemotherapy in esophageal cancer. J Nucl Med. 2011;52:369-78.

18. Molina D, Pérez-Beteta J, Luque B, Arregui E, Calvo M, Borrás $\mathrm{JM}$ et al. Tumor heterogeneity in glioblastoma assessed by MRI texture analysis: a potential marker of survival. Br J Radiol. 2016; 89:20160242.

19. Yoon H, Kim Y, Kim BS. Intratumoral metabolic heterogeneity predicts invasive components in breast ductal carcinoma in situ. Eur Radiol. 2015;12:3648-58.

20. García-Vicente AM, Soriano-Castrejón A, Relea-Calatayud F, Palomar-Muñoz A, León-Martín AA, Chacón-López-Muñiz I, et al. 18-F fluorodeoxyglucose retention index and biological prognostic parameters in breast cancer. Clin Nucl Med. 2012;37:470-6.

21. O'Connor J, Rose CJ, Waterton JC, Carano RA, Parker GJ, Jackson A. Imaging intratumor heterogeneity: role in therapy response, resistance, and clinical outcome. Cancer Res. 2014;21:249-57.

22. Mena E, Sheikhbahaei S, Taghipour M, Jha AK, Vicente E, Xiao $\mathrm{J}$, et al. 18F-FDG PET/CT metabolic tumor volume and intratumoral heterogeneity in pancreatic adenocarcinomas. Impact of dual-time point and segmentation methods. Clin Nucl Med. 2017;42:e16-21.

23. Lambin P, Rios-Velazquez E, Leijenaar R, Carvalho S, van Stiphout RG, Granton P, et al. Radiomics: extracting more information from medical images using advanced feature analysis. Eur J Cancer. 2012;48:441-6.

24. Brooks FJ, Grigsby PW. The effect of small tumor volumes on studies of intratumoral heterogeneity of tracer uptake. J Nucl Med. 2014;55:37-42.

25. Hatt M, Tixier F, Rest CLC, Pradier O, Visvikis D. Robustness of intratumour 18F-FDG PET uptake heterogeneity quantification for therapy response prediction in oesophageal carcinoma. Eur $\mathbf{J}$ Nucl Med Mol Imaging. 2013;40:1662-71.

26. Hatt M, Majdoub M, Vallières M, Tixier F, Le Rest CC, Groheux D, et al. 18F-FDG PET uptake characterization through texture analysis: investigating the complementary nature of heterogeneity and functional tumor volume in a multi-cancer site patient cohort. J Nucl Med. 2015;56:38-44.

27. Molina D, Pérez-Beteta J, Martínez-González A, Martino J, Velásquez C, Arana E, et al. Influence of gray-level and space discretization on brain tumor heterogeneity measures obtained from magnetic resonance images. Comput Med Biol. 2016;78:49-57.

28. Son SH, Kim DH, Hong CM, Kim CY, Jeong SY, Lee SW, et al. Prognostic implication of intratumoral metabolic heterogeneity in invasive ductal carcinoma of the breast. BMC Cancer. 2014;14:585-96.

29. Groheux D, Majdoub M, Tixier F, Le Rest CC, Martineau A, Merlet P, et al. Do clinical, histological or immunohistochemical primary tumour characteristics translate into different (18)F-FDG $\mathrm{PET} / \mathrm{CT}$ volumetric and heterogeneity features in stage II/III breast cancer? Eur J Nucl Med Mol Imaging. 2015;42:1682-91. 